\title{
BMJ Open Postsurgical rehabilitation for adults with low back pain with or without radiculopathy who were treated surgically: protocol for a mixed studies systematic review
}

\author{
Carol Cancelliere (D) ,, Jessica J. Wong, ${ }^{2,3}$ Hainan Yu, ${ }^{1,2}$ Margareta Nordin, ${ }^{4}$ \\ Silvano Mior (ID , 2,5 Paulo Pereira, 6,7 Ginny Brunton,, ${ }^{1,8}$ Heather Shearer, 2,9 \\ Gaelan Connell, ${ }^{2,10}$ Leslie Verville, ${ }^{1,2}$ Anne Taylor-Vaisey, ${ }^{1,2}$ Pierre Côté ${ }^{1,2}$
}

To cite: Cancelliere C, Wong JJ, Yu $\mathrm{H}$, et al. Postsurgical rehabilitation for adults with low back pain with or without radiculopathy who were treated surgically: protocol for a mixed studies systematic review. BMJ Open 2020;10:e036817. doi:10.1136/ bmjopen-2020-036817

- Prepublication history and additional material for this paper are available online. To view these files, please visit the journal online (http://dx.doi org/10.1136/bmjopen-2020036817).

Received 05 January 2020 Revised 18 February 2020 Accepted 06 March 2020
Check for updates

(C) Author(s) (or their employer(s)) 2020. Re-use permitted under CC BY-NC. No commercial re-use. See rights and permissions. Published by BMJ.

For numbered affiliations see end of article.

Correspondence to Dr Carol Cancelliere; carolina.cancelliere@uoit.ca

\section{ABSTRACT}

Introduction Surgical rates for low back pain (LBP) have been increasing in Europe, North America and Asia. Many patients treated surgically will require postsurgical rehabilitation. Little is known about the effectiveness of postsurgical rehabilitation interventions on health outcomes or about patients' experiences with these interventions.

Objectives To conduct a mixed studies systematic review of quantitative and qualitative studies regarding: (1) the effectiveness and safety of postsurgical rehabilitation interventions for adults with LBP treated surgically and (2) the experiences of patients, healthcare providers, caregivers or others involved with the rehabilitation. Methods and analysis We will search MEDLINE, Embase, PsycINF0, CINAHL, the Index to Chiropractic Literature, the Cochrane Controlled Register of Trials and the Rehabilitation \& Sports Medicine Source for peerreviewed empirical studies published from inception in any language. Studies using quantitative, qualitative and mixed methodologies will be included. We will also search reference lists of all eligible articles. Data extraction will include type of presurgical pathology, indication for surgery, surgical procedure, how the intervention was delivered and by whom, context and setting. We will conduct a quality assessment of each study and consider study quality in our evidence synthesis. We will use a sequential approach at the review level to synthesise and integrate data. First, we will synthesise the quantitative and qualitative studies independently, conducting a metaanalysis of the quantitative studies if appropriate and thematic synthesis of the qualitative studies. Then, we will integrate the quantitative and qualitative evidence by juxtaposing the findings in a matrix.

Ethics and dissemination Ethical approval is not required for this knowledge synthesis. Findings will be disseminated through knowledge translation activities including: (1) presentations at national and international conferences and scientific meetings; (2) presentations to local and international stakeholders; (3) publications in peer-reviewed journals and (4) posts on organisational websites.

\section{Strengths and limitations of this study}

- This is the first systematic review of the quantitative and qualitative literature to examine the effectiveness and safety of postsurgical rehabilitation interventions in adults with low back pain with or without radiculopathy who were treated surgically.

- This review will consider studies with a broad range of rehabilitation interventions as described by WHO, and outcomes as described by the International Classification of Functioning, Disability and Health framework.

- This systematic review protocol follows the Preferred Reporting Items for Systematic Review and MetaAnalysis Protocols guidelines.

- This review has no language limitation in articles.

- This review is limited to published, peer-reviewed articles, and potential publication bias will not be assessed.

PROSPERO registration number CRD42019134607.

\section{INTRODUCTION}

Rationale

Low back pain (LBP) is burdensome to patients, their families, healthcare systems and society. ${ }^{1}$ LBP is the leading cause of years lived with disability globally, and may be associated with falls, occupational injuries or motor vehicle collisions. ${ }^{2-7}$ It is a common condition, with a lifetime prevalence estimated at approximately $38.9 \%$ globally. ${ }^{8}$ LBP may present with radiculopathy, whereby the patient experiences pain, weakness or numbness in the leg due to irritation of spinal nerve roots (eg, from foraminal stenosis or disc herniation).${ }^{9}{ }^{10}$ LBP is also associated with high healthcare costs and frequent healthcare use. ${ }^{5-7}$ Although most episodes 
resolve, approximately $20 \%$ of adults with LBP experience chronic symptoms, functional limitations or difficulties participating in their communities or returning to work. ${ }^{11} 12$

A proportion of patients suffering from LBP undergo surgery, particularly those with persistent radicular symptoms. In the UK (population of about 63 million), approximately $10 \quad 000$ lumbar disc excisions were performed in hospitals of the National Health Service from 2011 to $2012 .{ }^{13}{ }^{14}$ In the USA (population of about 287 million), approximately 280000 lumbar discectomies were performed in 2002. ${ }^{15}$ Surgical rates for these conditions have also increased over time. In England, the number of surgical procedures for degenerative lumbar spine disease nearly doubled between 1999 and 2013 (ie, from 25 to 49 per 100000$).{ }^{16}$ Similar trends have been reported in other regions, including in Asia and the USA. $^{17-22}$ The surgical rates for lumbar disc herniations have also increased over time. For instance, the number of lumbar disc surgeries increased twofold in South Korea from 2003 to $2008 .^{23}$

Postsurgical rehabilitation refers to interventions provided following surgery, and is often recommended to patients who undergo lumbar spinal surgery. It is aimed at helping individuals, who are experiencing or likely to experience disability, achieve and maintain optimal functioning within their environments. ${ }^{24}$ Rehabilitation programs can target improvements in individual functioning (eg, exercises, non-pharmacological or pharmacological therapies) or the individual's environment (eg, assistive devices, adaptations to the workplace). However, little is known about the effectiveness of postsurgical rehabilitation in managing patients' symptoms, function and return to activity. Few systematic reviews have synthesised the evidence in this area to better inform shared decision making related to postsurgical rehabilitation for LBP. A previous systematic review ${ }^{25}$ examining the effectiveness of rehabilitation following lumbar disc surgery suggested that exercise programs starting 4-6 weeks postsurgery led to a faster decrease in pain and disability compared with no treatment. Better results were seen with highintensity vs low-intensity exercise. ${ }^{25}$ However, the authors noted that the overall quality of the evidence was low, and high-quality randomised trials were needed. ${ }^{25}$ In another systematic review ${ }^{26}$ that examined the effectiveness of rehabilitation following first-time lumbar disc surgery, authors reported strong evidence for considering intensive exercise programs 4-6 weeks following surgery. In two highquality randomised controlled trials (RCTs), ${ }^{27} 28$ intensive exercise programs were more effective in improving functional status and led to faster return to work during shortterm follow-up (6 months) compared with mild exercise programs. However, there was no difference between intensive exercise programs and mild exercise programs with regard to improvement at the 12-month follow-up. Finally, evidence from a systematic review ${ }^{29}$ examining the effectiveness of rehabilitation for lumbar stenosis indicated that active rehabilitation following decompression surgery was more effective than usual care in improving both short-term and long-term functional status. ${ }^{29}$

Healthcare providers implementing evidence-based postsurgical rehabilitation should also consider its effects on patient-important outcomes, such as return to work or meaningful activities. These outcomes should be informed by the International Classification of Functioning, Disability and Health (ICF) framework, ${ }^{30}$ and by the experiences of those involved in rehabilitation such as patients, healthcare providers and caregivers. Additionally, little is known about individuals' experiences and perceptions about spine surgery and postsurgical rehabilitation. ${ }^{31-33}$ Primary studies of patient experience provide a number of overlapping themes. Abbott et al explored patient experiences postlumbar fusion within the context of the ICF framework. ${ }^{30}$ Patients' experiences were most frequently linked to psychological, sensory, neuromusculoskeletal and movement attributes, which correspond to the 'body function and structures' and 'activity' domains of the ICF framework. ${ }^{30}$ Archer et al identified several factors based on patient interviews that could optimise recovery. ${ }^{31}$ These include an ability to adjust expectations, acceptance of functional outcomes, setting realistic and achievable goals, provision of familial and friend support, being positive and realistic, working hard at rehabilitation and communicating with caregivers. ${ }^{31}$ Rushton et al used focus group methodology to explore perceptions, preferences and feelings of the rehabilitation programme in patients who underwent lumbar discectomy and physiotherapy. ${ }^{32}$ They found variation in patients' preferences for leaflet only, individual one-on-one or group physiotherapist led interventions, suggesting that a stepped care approach accounting for patient preferences may be of value when planning postsurgical spine rehabilitation programs. ${ }^{32}$ Therefore, understanding patients' experiences and preferences with postsurgical care, as well as healthcare providers and other caregivers, may help explain the outcomes of postsurgical rehabilitation interventions and improve programme compliance and recovery rates. ${ }^{34}$

The previous systematic reviews have limitations and require updating. For example, the literature searches for the systematic reviews published in 2014 and 2003 ended in 2013 and 2000, respectively. ${ }^{25} 2629$ Some systematic reviews included randomised trials only, and thus may not have captured evidence from observational and qualitative studies potentially useful for informing programs and policy. ${ }^{25}{ }^{29}$ We are not aware of any systematic reviews of qualitative studies exploring experiences with postsurgical spine rehabilitation among individuals involved in rehabilitation. Qualitative evidence can help to illuminate the mechanisms through which interventions work. ${ }^{35}$ Finally, the previous reviews focused on clinical rehabilitation. Our review will investigate rehabilitation from a clinical and community-based perspective, explore patients' and others' experiences and perspectives with such interventions, and then integrate these results into a unique practical matrix. To our best knowledge, there 
are no systematic reviews examining the effectiveness of a wide range of postsurgical rehabilitation interventions (including community-based rehabilitation) in adults treated surgically for LBP. Furthermore, there are no reviews that combine the findings from different types of studies to produce a more comprehensive synthesis of the evidence on 'what works' for this population.

\section{Objectives}

Our overall objective is to systematically search, assess and synthesise quantitative and qualitative studies regarding the effectiveness and experiences of postsurgical rehabilitation interventions for improving pain, health, functioning and disability among adults with LBP treated surgically. Our review questions are:

1. What is the effectiveness and safety of postsurgical rehabilitation interventions for improving pain, function, disability and health outcomes in adults with LBP with or without radiculopathy who were treated with surgery?

2. What are patients', providers' and caregivers' experiences, views and opinions of postsurgical rehabilitation interventions delivered in any healthcare setting for adults with LBP with or without radiculopathy who were treated with surgery?

3. What can be hypothesised from the integration of the quantitative and qualitative evidence about the effectiveness and safety of postsurgical rehabilitation interventions in adults with LBP with or without radiculopathy who were treated with surgery?

We are targeting healthcare professionals and policy makers involved in delivering and planning postsurgical rehabilitation interventions. We aim to provide them with knowledge regarding effective and positively experienced interventions for adults treated surgically for LBP.

\section{METHODS}

We developed this systematic review protocol using the Preferred Reporting Items for Systematic Reviews and Meta-Analyses for Protocols ${ }^{36}$ (online supplementary file 1). ${ }^{37}$ We will report our systematic review according to the Preferred Reporting Items for Systematic Reviews and Meta-Analyses statement. ${ }^{38}$

\section{Eligibility criteria}

\section{Population}

We targeted studies including adults (aged 18 years and older) who underwent surgery for LBP with or without radiculopathy. We defined LBP as pain and discomfort below the costal margin and above the inferior gluteal folds, with or without referred leg pain. ${ }^{39}$ Radiculopathy refers to inflammation, injury/dysfunction or compression of the spinal nerve roots that can present as pain, weakness or altered sensation in a myotomal or dermatomal distribution. Lumbar radiculopathy may be attributed to spinal stenosis (narrowing of the spinal or foraminal canals) or lumbar disc herniation (localised displacement of disc material beyond the normal margins of the intervertebral disc space)..$^{10} 40$

\section{Intervention}

The quantitative component of this review included studies that investigated the effectiveness of postsurgical spine rehabilitation interventions or programs of care initiated within the first 3 months after surgery. The WHO defines rehabilitation as a set of interventions that assist individuals who experience, or are likely to experience, disability to achieve and maintain optimal functioning when interacting with their environments. ${ }^{24}$

Postsurgical rehabilitation interventions include rehabilitation medicine/therapy, which aims to: (1) improve function through the diagnosis and treatment of health conditions, reducing impairments, preventing or treating complications and (2) restore and compensate loss of functioning, and prevent or slow deterioration in functioning in every area of a person's life. ${ }^{24}$ Rehabilitation interventions may also include assistive technology, which refers to any item, piece of equipment, or product used to increase, maintain or improve functional capabilities. ${ }^{24}$ Postsurgical rehabilitation interventions can be provided by different healthcare providers including, but not limited to, general practitioners, orthopaedic surgeons, physiotherapists, chiropractors and occupational therapists. Examples of postsurgical rehabilitation interventions include, but are not limited to (table 1):

1. Patient education and self-management.

2. Compensatory strategies, training and exercises (eg, stretching, strengthening, range of motion; supervised or unsupervised; aerobic or anaerobic exercises).

3. Manual therapies (eg, manipulation, mobilisation, traction, soft tissue therapy).

4. Passive physical modalities (eg, ultrasound, interferential current therapy, transcutaneous electrical nerve stimulation).

5. Acupuncture (eg, needle acupuncture, electroacupuncture, acupressure).

6. Pharmacological interventions used in the rehabilitation period (ie, not intended to alleviate procedural/surgery-related pain) (eg, non-steroidal anti-inflammatory drugs, acetaminophen, muscle relaxants, routes of administration include topical, oral or injected).

7. Social support and advice.

8. Psychological interventions (eg, cognitive behavioural therapy).

9. Modifications to the environment. ${ }^{414}$

10. Provision of resources.

11. Adaptation of workplace.

12. Assistive technologies (eg, crutches, orthoses, braces or wheelchairs for people with mobility impairments).

\section{Comparators}

The quantitative component of this review considered comparisons including other interventions, placebo or sham interventions, wait list and no intervention. 
Table 1 Examples of selected interventions for rehabilitation

\begin{tabular}{|c|c|c|}
\hline Intervention & Definition & Examples \\
\hline $\begin{array}{l}\text { Patient education and self- } \\
\text { management }\end{array}$ & $\begin{array}{l}\text { Teaching patients skills that they can use to manage } \\
\text { their health condition }\end{array}$ & $\begin{array}{l}\text { Learning disease-specific information } \\
\text { Learning general managing skills (eg, problem } \\
\text { solving, finding and using community resources, } \\
\text { working with healthcare team) } \\
\text { Learning strategies to increase confidence (ie, } \\
\text { self-efficacy) in ability to engage in behaviours } \\
\text { that are needed to manage their condition on a } \\
\text { daily basis } \\
\text { Adequate peer role models and support networks } \\
\text { that facilitate the initiation and maintenance of } \\
\text { desired behavioural changes }\end{array}$ \\
\hline Exercise & $\begin{array}{l}\text { A subcategory of physical activity that is planned, } \\
\text { structured, repetitive and purposeful; can be } \\
\text { supervised (eg, by a healthcare professional) or } \\
\text { unsupervised }\end{array}$ & $\begin{array}{l}\text { Stretching } \\
\text { Strengthening } \\
\text { Range of motion exercises } \\
\text { Aerobic (eg, swimming, cycling, walking, running) } \\
\text { Anaerobic (eg, jumping, sprinting, weight lifting) }\end{array}$ \\
\hline & $\begin{array}{l}\text { Traction: manual or mechanically assisted application } \\
\text { of an intermittent or continuous distractive force }\end{array}$ & \\
\hline & $\begin{array}{l}\text { Soft tissue therapy: a mechanical form of therapy } \\
\text { where soft-tissue structures are pressed and kneaded, } \\
\text { using physical contact with the hand or mechanical } \\
\text { device }\end{array}$ & \\
\hline Passive physical modalities & $\begin{array}{l}\text { A form of cold, heat or light application affecting the } \\
\text { body at the skin level or ultrasonic or electromagnetic } \\
\text { radiation affecting structures beneath the skin surface: } \\
\text { Passive assistive devices: device to encourage } \\
\text { immobilisation in anatomic positions or actively inhibit } \\
\text { or prevent movement }\end{array}$ & $\begin{array}{l}\text { Heat application: heat pack, hydrotherapy } \\
\text { Cryotherapy: cold pack, vapocoolant spray } \\
\text { Low-level laser } \\
\text { Electrical muscle stimulation } \\
\text { - Pulsed electromagnetic therapy }\end{array}$ \\
\hline
\end{tabular}

\begin{tabular}{|c|c|c|}
\hline Acupuncture & $\begin{array}{l}\text { Any body needling, moxibustion, electric acupuncture, } \\
\text { laser acupuncture, microsystem acupuncture and } \\
\text { acupressure }\end{array}$ & $\begin{array}{l}\text { Traditional needling } \\
\text { Dry needling } \\
\text { Burning of specific herbs } \\
\text { Electroacupuncture } \\
\text { Photoacupuncture }\end{array}$ \\
\hline Pharmacological interventions & A substance used in treating disease or relieving pain & $\begin{array}{l}\text { Acetaminophen } \\
\text { Nonsteroidal anti-inflammatory drugs } \\
\text { Muscle relaxants } \\
\text { Antidepressants }\end{array}$ \\
\hline Assistive technologies & $\begin{array}{l}\text { Any item, piece of equipment or product system, } \\
\text { used to increase, maintain or improve the functional } \\
\text { capabilities of people with disabilities }\end{array}$ & $\begin{array}{l}\text { Walking aids } \\
\text { Orthoses } \\
\text { Braces } \\
\text { Wheelchairs }\end{array}$ \\
\hline
\end{tabular}

\section{Outcomes}

The quantitative component of this review considered studies that included outcomes related to pain, functioning, disability and health as described by the ICF framework, as well as adverse events (table 2). ${ }^{43}$ These will include:
1. Body function and structure (to describe a person's impairment).

a. Examples of constructs include: pain (eg, intensity, frequency, duration), range of motion, psychological and experiential outcomes (eg, depression, anxiety). 
Table 2 Definitions from the International Classification of Functioning, Disability and Health

\begin{tabular}{|c|c|}
\hline Term & Definition \\
\hline $\begin{array}{l}\text { Body } \\
\text { functions }\end{array}$ & $\begin{array}{l}\text { Physiological functions of body systems } \\
\text { (including psychological functions) }\end{array}$ \\
\hline $\begin{array}{l}\text { Body } \\
\text { structures }\end{array}$ & $\begin{array}{l}\text { Anatomical parts of the body such as } \\
\text { organs, limbs and their components }\end{array}$ \\
\hline Impairments & $\begin{array}{l}\text { Problems in body function or structure such } \\
\text { as a significant deviation or loss }\end{array}$ \\
\hline Activity & Execution of a task or action by an individual \\
\hline Participation & Involvement in a life situation \\
\hline $\begin{array}{l}\text { Activity } \\
\text { limitations }\end{array}$ & $\begin{array}{l}\text { Difficulties an individual may have in } \\
\text { executing activities }\end{array}$ \\
\hline $\begin{array}{l}\text { Participation } \\
\text { restrictions }\end{array}$ & $\begin{array}{l}\text { Problems an individual may experience in } \\
\text { involvement in life situations }\end{array}$ \\
\hline $\begin{array}{l}\text { Environmental } \\
\text { factors }\end{array}$ & $\begin{array}{l}\text { External contextual factors that make up the } \\
\text { physical, social and attitudinal environment } \\
\text { in which people live and conduct their lives }\end{array}$ \\
\hline $\begin{array}{l}\text { Personal } \\
\text { factors }\end{array}$ & $\begin{array}{l}\text { Internal contextual factors that influence } \\
\text { how disability is experienced by the } \\
\text { individual }\end{array}$ \\
\hline
\end{tabular}

b. Examples of tools to measure constructs: Numeric Rating Scale or Visual Analogue Scale to measure pain intensity ${ }^{44-46}$; goniometer to measure range of motion; Centre for Epidemiologic Studies Depression Scale ${ }^{47}$ or Beck Depression Inventory to measure depressive symptoms. ${ }^{48} 49$

2. Activity and participation (to describe a person's functional status, health-related quality of life).

a. Examples include disability, communication, mobility, interpersonal interactions, preferences, selfcare, learning, applying knowledge, return to work/ activities/school.

b. Examples of tools to measure constructs: RolandMorris Low Back Pain and Disability Questionnaire ${ }^{39}$ or Oswestry Disability Index to measure disability ${ }^{50}$; 36-item Short Form Survey (SF-36) ${ }^{51}$ or EuroQol-5D (EQ-5D $)^{52}$ for quality of life.

3. Additional surgeries.

a. Examples include revision surgeries, surgeries for complications.

4. Adverse events.

We define adverse events as any unfavourable sign, symptom or disease temporarily associated with the treatment, whether or not caused by the treatment. ${ }^{53}$ We will also consider indirect harms, where the use of an intervention delays a diagnosis or treatment, and such delay holds a potential harm. ${ }^{54}$

\section{Phenomena of interest}

The qualitative component of this review considered studies that explore the experiences, views and opinions (eg, perceived benefits and challenges) regarding postsurgical rehabilitation interventions related to surgery for LBP with or without radiculopathy among patients, healthcare providers, caregivers and others involved with postsurgical rehabilitation.

\section{Study design}

We will conduct a mixed studies review. ${ }^{55}$ This design integrates quantitative, qualitative and mixed methods studies, providing a rich, detailed understanding of complex health interventions and programs. ${ }^{56} \mathrm{We}$ included studies published in peer-reviewed journals and representing a range of methodologies: randomised controlled studies (minimum 30 participants per arm at baseline), cohort studies and case-control studies (minimum 100 participants per group at baseline), qualitative and mixed method studies. A sample size of 30 in randomised controlled studies is conventionally considered the minimum needed for non-normal distributions to approximate the normal distribution. ${ }^{57}$ The assumption that data are normally distributed is required to ascertain a difference in sample means between treatment arms. In cohort and case-control studies, a sample of 100 is conventionally considered the minimum needed to obtain well-balanced groups at baseline and control bias (especially confounding by indication). Qualitative studies included phenomenology, grounded theory, ethnography, action research and descriptive qualitative studies, or any qualitative methodologies that explore patients', providers' or others' experiences with postsurgical rehabilitation related to surgery for LBP with or without radiculopathy. Mixed methods studies were only considered if data from the quantitative or qualitative components could be clearly extracted.

\section{Context and setting}

We considered postsurgical rehabilitation interventions/ programs of care delivered in any healthcare organisation within a metropolitan or non-metropolitan (regional, rural and remote) area and in any healthcare setting (eg, acute care, hospital, primary healthcare, rehabilitation clinics), or in the community. Community-based rehabilitation is implemented through the combined efforts of people with disabilities, their families and communities, relevant government and non-government health, education, vocational, social and other services (eg, workplace interventions, advocacy programme)..$^{58}$

\section{Exclusion criteria}

We excluded: (1) studies of patients with LBP with or without radiculopathy attributed to major structural or systemic pathology (eg, fracture, infection, tumour, osteoporosis, inflammatory arthritides and cauda equina syndrome); (2) studies of patients with failed back surgery syndrome defined as surgical end-stage after one or several surgical interventions to relieve LBP with or without radiculopathy without positive effect $\mathrm{t}^{59}$; (3) studies assessing pharmacological interventions for procedural pain control alone immediately postsurgery; (4) studies assessing postsurgical rehabilitation interventions solely conducted at the societal level, such as barrier removal 
initiatives (eg, fitting a ramp to a public building); (5) study designs including pilot studies assessing feasibility, protocol studies, cross-sectional studies, case reports, case series, systematic reviews and other review papers, clinical practice guidelines, biomechanical studies, laboratory studies, cadaveric or animal studies and conceptual papers and (6) publication types including letters, editorials, commentaries, unpublished manuscripts, dissertations, government reports, books and book chapters, conference proceedings, meeting abstracts, lectures and addresses, consensus development statements and guideline statements.

\section{Information sources}

We developed the search strategy in consultation with an experienced health sciences librarian. The search was reviewed by a second experienced health sciences librarian using the Peer Review of Electronic Search Strategies Checklist. ${ }^{6061}$ We conducted an electronic search of the following databases from database inception: MEDLINE (Ovid), Embase (Ovid), PsycINFO (Ovid), CINAHL (Cumulative Index to Nursing and Allied Health Literature, EBSCO host), the Index to Chiropractic Literature (Chiropractic Library Collaboration), the Cochrane Controlled Register of Trials (Ovid) and the Rehabilitation \& Sports Medicine Source (EBSCOhost). We will also search the reference lists of all eligible articles for additional relevant studies. We included studies in any language and will use professional medical translation services where required.

\section{Search strategy}

The searches included a combination of subject headings specific to databases (eg, MeSH in MEDLINE) and free text words to capture the key concepts of postsurgical rehabilitation and LBP with or without radiculopathy (online supplementary file 2).

\section{Patient and public involvement}

Patients were not involved with the design of our study. However, our research questions and outcomes stemmed from our collective clinical experiences and encounters with patients with LBP who received surgery. We also included qualitative studies with an aim of capturing patients' and caregivers' experiences and preferences regarding postsurgical interventions. Public consultation will be conducted during the guidelines development phase of this project.

\section{STUDY RECORDS}

\section{Data management}

Electronic search results were downloaded into Endnote X9 reference manager software (Clarivate Analytics, Philadelphia, Pennsylvania, USA). We removed duplicates and the remaining references were uploaded to the Evidence for Policy and Practice Information (EPPI) and Coordinating Centre Reviewer software for the data extraction stages (EPPI-Reviewer V.4, UCL Institute of Education, University of London, UK). EPPI-Reviewer software stores references, manages and monitors the data extraction process and provides an audit trail for the review. ${ }^{62}$

\section{Selection process}

First, paired reviewers independently screened titles and abstracts for assessment against the inclusion and exclusion criteria. Second, the reviewers independently assessed the full text of each selected article to confirm its inclusion in the study. Disagreements related to the inclusion of any article were discussed and resolved, involving a third reviewer if necessary. We conducted training exercises prior to initiating the screening process to ensure high inter-rater reliability. Review members have been meeting weekly to discuss the progress of the screening process and any unanticipated problems.

\section{Data items and data extraction process}

Paired reviewers will independently extract the data from all eligible studies. The data extracted will include details about: (1) populations: sociodemographic information (eg, age, sex, gender, education, occupation, culture), health status (eg, comorbidities, previous surgeries), geographical region, type of presurgical pathology (eg, herniated nucleus pulposus, spondylolisthesis and spinal stenosis), indication for surgery (eg, acute/subacute/ chronic LBP with or without radiculopathy), surgical procedure (eg, microdiscectomy, decompressive laminectomy/laminotomy/foraminotomy, spinal fusion); (2) study methods; (3) interventions: type (eg, resistance exercise, manual therapy, modalities), setting (eg, hospital, community), temporality (time of initiation after surgery), type of healthcare provider(s) delivering intervention, duration (eg, 4 weeks, 3 months), dosage (eg, 3 times per week); (4) comparators; (5) phenomena of interest or outcomes of significance to the review objective linked to corresponding ICF categories (body functions and structures, activity and participation, and environmental and personal factors) by established linking rules, ${ }^{63-65}$ additional surgeries and adverse events; (6) key findings stratified according to patient characteristics, presurgical pathology, type of surgical intervention and specific type of postsurgical rehabilitation therapies or programs of care, where possible and (7) methodological quality of studies. If multiple outcome measures are used to assess one construct, we will stratify our analyses by outcome measures and describe how the results vary. We will report the unit of analysis of adverse events as reported by the study authors (eg, proportion of participants that experienced adverse events or number of adverse events experienced). Paired reviewers will pretest the data extraction form and revise as needed. EPPI-reviewer software will be used to manage the data extraction process.

Any disagreements that arise between the reviewers will be resolved through discussion or with a third reviewer. 
Authors of papers will be contacted to request missing or additional data, where required.

\section{Quality assessment}

We will assess the quality of studies using the Scottish Intercollegiate Guidelines Network (SIGN) criteria for RCTs, cohort studies and case-control studies ${ }^{66}$; the Joanna Briggs Institute (JBI) critical appraisal checklist for qualitative studies ${ }^{67}$ and the Mixed Methods Appraisal Tool (MMAT) for mixed methods studies. ${ }^{68}$ The SIGN checklists allow reviewers to assess internal validity by considering the impact of selection bias, information bias and confounding on study results. The JBI checklist allows reviewers to assess the possibility of bias in qualitative studies' design, conduct and analysis. The MMAT allows reviewers to assess the interdependent qualitative and quantitative components of the study and criteria to consider, such as justification for mixing evidence, and appropriate ways of integrating the data. We will categorise the validity or credibility of each study as either acceptable or unacceptable. Authors of papers will be contacted to request missing or additional data for clarification where required. Paired reviewers will independently assess the selected studies for quality. Any disagreements that arise between the reviewers will be resolved through discussion or with a third reviewer.

\section{DATA}

\section{Data synthesis}

We will use a sequential approach at the review level to synthesise and integrate the data. ${ }^{35}$ This will involve separate quantitative and qualitative synthesis followed by integration of the resultant quantitative and qualitative evidence. We will interpret the findings in consideration of study quality.

\section{Quantitative synthesis}

Clinical, methodological and statistical (using the $\mathrm{I}^{2}$ statistic) ${ }^{69}$ heterogeneity among studies will be assessed. Clinical heterogeneity may result from differences in populations, interventions, comparators or outcomes across studies. Methodological and statistical heterogeneity may result from differences in risk of bias and differences in outcomes across studies beyond what could be expected by chance alone. Methodological heterogeneity across studies will be assessed based on the overall judgement from SIGN as low or moderate (acceptable) vs high (unacceptable) risk of bias. Statistical heterogeneity will be assessed using the $\mathrm{I}^{2}$ statistic, whereby $\mathrm{I}^{2}$ of $<25 \%-$ $50 \%$ will be considered low to moderate (homogeneous) and $\geq 50 \%$ considered high (heterogeneous). ${ }^{69}$ Where possible, data will be pooled with statistical meta-analysis using EPPI-Reviewer software. Where statistical pooling is not possible (ie, if there is clinical, methodological and statistical heterogeneity), the findings will be presented qualitatively according to the principles of best evidence synthesis. ${ }^{70}$ We will use data provided in the studies to measure the association between tested interventions and outcomes by computing the relative risk and its $95 \%$ CI where this information is available. Similarly, we will compute the difference in mean change between groups and $95 \%$ CI to quantify the effectiveness of interventions. The computation of the $95 \%$ CI for the difference in mean change is based on the assumption that the preintervention and postintervention outcomes are highly correlated $(\mathrm{r}=0.8) .{ }^{71}{ }^{72}$ We will interpret the evidence on the effectiveness of postsurgical rehabilitation interventions by determining whether an intervention was superior, equal or inferior to a comparison intervention.

\section{Qualitative synthesis}

We will use thematic synthesis to synthesise the qualitative research findings. ${ }^{7374}$ First, verbatim study findings will be entered into a database (EPPI-Reviewer). Pairs of trained reviewers will then independently code each line of text and develop descriptive themes according to its meaning and content, and subsequently generate analytical themes to answer our review questions. Reviewers will finalise the descriptive and analytical themes through discussion.

\section{Integration of quantitative and qualitative evidence}

We will juxtapose findings using a matrix to compare and contrast the findings across the syntheses in the review. The use of a matrix will allow us to explore heterogeneity in the findings of the quantitative studies and may indicate why some interventions may be effective and safe, and some may not. ${ }^{35}$ For example, we may list themes from the qualitative synthesis along one side of the matrix, and then plot the interventions evaluated in the quantitative synthesis against the themes as either a match (when the intervention matched a theme) or a mismatch (when the intervention was the opposite of a theme). We will identify gaps in knowledge if a particular theme for an intervention does not match with any of the interventions evaluated in the quantitative studies.

\section{DISSEMINATION}

We will collaborate with our national and international consultants and knowledge users, who include decision makers and managers, healthcare and other service providers, patients and their caregivers. In addition to this mixed studies review, we will provide clinical scenarios and demonstrate how healthcare providers and others can apply this evidence in an effort to improve patient health, function and disability outcomes after LBP surgery. Knowledge translation activities will include ongoing consultation and feedback between knowledge users and the research team during preparation of our project deliverables; presentations in national and international conferences and scientific meetings; presentations to local and international stakeholders; publications in peer-reviewed journals; posts and lay language summaries on organisations' websites (open access). 


\section{DISCUSSION}

Findings from this mixed studies review will advance our knowledge on the effectiveness and safety of a wide range of postsurgical rehabilitation interventions for LBP, as well as improve our understanding of patients' and other key individuals' experiences and perceptions of rehabilitation interventions. This work will provide the evidentiary basis to develop user-friendly care pathways outlining evidence-based postsurgical rehabilitation, which can be adapted for specific settings (eg, hospitals, rehabilitation clinics and workplaces) and geographical regions prior to implementation. Specifically, postsurgical rehabilitation interventions that are identified as effective, safe and positively experienced could be considered by patients, their families and caregivers, healthcare providers and other stakeholders (eg, workplaces) during shared decision making. In contrast, interventions that are identified as ineffective (eg, leading to outcomes no better than comparison interventions) or even harmful, and negatively experienced, should not be considered when planning the postsurgical rehabilitation programme of care. Moreover, we will map findings to the ICF framework to allow healthcare providers and stakeholders to use standardised language in the assessment and management of individuals during their postsurgical rehabilitation programme. This may further facilitate improvements in postsurgical functioning, disability and health outcomes in this patient population.

Our review has potential limitations. We included only published, peer-reviewed studies; therefore, publication bias is possible. Our literature search strategies may have missed potentially relevant studies. However, we tried to mitigate this by having a second experienced health sciences librarian peer review the searches, and searching reference lists of eligible studies. Finally, we excluded RCTs with fewer than 30 participants per treatment arm. This may have omitted potentially relevant studies; however, this minimum sample size is recommended to apply central limit theorem for non-normal distributions to approximate normal distributions. ${ }^{57}$

Findings from our review will guide future research by identifying both the methodological limitations of previous studies, and the knowledge gaps in the current scientific literature. Future studies can be designed to minimise these methodological limitations and address key knowledge gaps to further advance our understanding of the role of postsurgical rehabilitation for patients with LBP. This novel interpretation of quantitative and qualitative evidence according to the ICF framework serves as a model for how outcomes related to pain, functioning, disability and health can be prioritised in future research. ${ }^{6}$

\footnotetext{
Author affiliations

${ }^{1}$ Faculty of Health Sciences, Ontario Tech University, Oshawa, Ontario, Canada

${ }^{2}$ Centre for Disability Prevention and Rehabilitation, Ontario Tech University and Canadian Memorial Chiropractic College, Oshawa, Ontario, Canada

${ }^{3}$ Epidemiology Division, Dalla Lana School of Public Health, University of Toronto, Toronto, Ontario, Canada
}

${ }^{4}$ Department of Orthopedic Surgery and Environmental Medicine, NYU School of Medicine, Occupational and Industrial Orthopedic Center, New York University, New York, New York, USA

${ }^{5}$ Research, Canadian Memorial Chiropractic College, Toronto, Ontario, Canada ${ }^{6}$ Spine Unit, Department of Neurosurgery, Centro Hospitalar Universitário São João, Porto, Portugal

${ }^{7}$ Faculty of Medicine, University of Porto, Porto, Portugal

${ }^{8}$ EPPI-Centre, UCL Institute of Education, University College London, London, UK ${ }^{9}$ Institute of Health Policy, Management and Evaluation, University of Toronto, Toronto, Ontario, Canada

${ }^{10}$ Rehabilitation Sciences, Faculty of Medicine, The University of British Columbia, Vancouver, British Columbia, Canada

Acknowledgements This research was undertaken, in part, thanks to funding from the Canada Research Chairs programme to Pierre Côté who holds the Canada Research Chair in Disability Prevention and Rehabilitation at Ontario Tech University, and from the Canadian Chiropractic Research Foundation to Carol Cancelliere who holds a Research Chair in Knowledge Translation in the Faculty of Health Sciences at Ontario Tech University.

Contributors CC, JJW and HY assisted in developing the research questions and systematic review methodology, drafted the manuscript, and reviewed and revised the manuscript.PC, MN, SM and PP assisted in developing the research questions and systematic review methodology, and reviewed and revised the manuscript. GB assisted in developing the systematic review methodology, and reviewed and revised the manuscript.HS, GC, LV and ATV assisted in developing the research questions and systematic review methodology, and reviewed and revised the manuscript.All authors approved the final manuscript.

Funding This work was supported by the Canadian Chiropractic Research Foundation and EUROSPINE TFR Grant. The funding organisations were not involved in the design or conduct of this systematic review protocol

Competing interests None declared.

Patient and public involvement Patients and/or the public were not involved in the design, or conduct, or reporting, or dissemination plans of this research. Refer to the Methods section for further details.

Patient consent for publication Not required.

Provenance and peer review Not commissioned; externally peer reviewed.

Open access This is an open access article distributed in accordance with the Creative Commons Attribution Non Commercial (CC BY-NC 4.0) license, which permits others to distribute, remix, adapt, build upon this work non-commercially, and license their derivative works on different terms, provided the original work is properly cited, appropriate credit is given, any changes made indicated, and the use is non-commercial. See: http://creativecommons.org/licenses/by-nc/4.0/.

\section{ORCID iDs}

Carol Cancelliere http://orcid.org/0000-0003-1883-4970

Silvano Mior http://orcid.org/0000-0001-6575-2797

\section{REFERENCES}

1 Hartvigsen J, Hancock MJ, Kongsted A, et al. What low back pain is and why we need to pay attention. Lancet 2018;391:2356-67.

2 Cassidy JD, Carroll LJ, Côté P. The Saskatchewan health and back pain survey. The prevalence of low back pain and related disability in Saskatchewan adults. Spine 1998;23:1860-6.

3 Cassidy JD, Côté P, Carroll LJ, et al. Incidence and course of low back pain episodes in the general population. Spine 2005;30:2817-23.

4 Hincapié CA, Cassidy JD, Côté $P$, et al. Whiplash injury is more than neck pain: a population-based study of pain localization after traffic injury. J Occup Environ Med 2010;52:434-40.

5 GBD 2015 Disease and Injury Incidence and Prevalence Collaborators. Global, regional, and national incidence, prevalence, and years lived with disability for 310 diseases and injuries, 19902015: a systematic analysis for the global burden of disease study 2015. Lancet 2016;388:1545-602.

6 Global Burden of Disease Study 2013 Collaborators. Global, regional, and national incidence, prevalence, and years lived with disability for 301 acute and chronic diseases and injuries in 188 countries, 19902013: a systematic analysis for the global burden of disease study 2013. Lancet 2015;386:743-800. 
7 Hoy D, March L, Brooks P, et al. The global burden of low back pain: estimates from the global burden of disease 2010 study. Ann Rheum Dis 2014;73:968-74.

8 Hoy D, Bain C, Williams G, et al. A systematic review of the global prevalence of low back pain. Arthritis Rheum 2012;64:2028-37.

9 The World Health Organization. Background paper 6.24: low back pain. Available: https://www.who.int/medicines/areas/priority_ medicines/BP6_24LBP.pdf [Accessed Dec 12 2018].

10 North American Spine Society. Nass clinical guidelines: lumbar disc herniation with radiculopathy. Available: https://www.spine.org/ Documents/ResearchClinicalCare/Guidelines/LumbarDiscHerniation. pdf [Accessed 12 Dec 2018].

11 Carey TS, Evans A, Hadler N, et al. Care-seeking among individuals with chronic low back pain. Spine 1995;20:312-7.

12 Carey TS, Evans AT, Hadler NM, et al. Acute severe low back pain. A population-based study of prevalence and care-seeking. Spine 1996;21:339-44.

13 Office for National Statistics. Table P07 2011 census: number of usual residents living in households and communal establishments, local authorities in England and Wales. Available: www.ons.gov. uk/ons/publications/re-reference-tables.html?edition=tcm\%3A77257414

14 The Health and Social Care Information Centre, Hospital Episode Statistics for England. Inpatient statistics, 2011-12. Available: www. hscic.gov.uk/hes01

15 Sherman J, Cauthen J, Schoenberg D, et al. Economic impact of improving outcomes of lumbar discectomy. Spine J 2010;10:108-16.

16 Sivasubramaniam V, Patel HC, Ozdemir BA, et al. Trends in hospital admissions and surgical procedures for degenerative lumbar spine disease in England: a 15-year time-series study. BMJ Open 2015;5:e009011.

17 Deyo RA, Mirza SK. Trends and variations in the use of spine surgery. Clin Orthop Relat Res 2006;443:139-46.

$18 \mathrm{Kim}$ P, Kurokawa R, Itoki K. Technical advancements and utilization of spine surgery--international disparities in trend-dynamics between Japan, Korea, and the USA. Neurol Med Chir 2010;50:853-8.

19 Pumberger M, Chiu Y-L, Ma Y, et al. National in-hospital morbidity and mortality trends after lumbar fusion surgery between 1998 and 2008. J Bone Joint Surg Br 2012;94:359-64.

20 Rajaee SS, Bae HW, Kanim LEA, et al. Spinal fusion in the United States: analysis of trends from 1998 to 2008. Spine 2012;37:67-76.

21 Weinstein JN, Lurie JD, Olson PR, et al. United States' trends and regional variations in lumbar spine surgery: 1992-2003. Spine 2006;31:2707-14.

22 Yoshihara H, Yoneoka D. National trends in the surgical treatment for lumbar degenerative disc disease: United States, 2000 to 2009. Spine J 2015;15:265-71.

$23 \mathrm{Kim} \mathrm{CH}$, Chung CK, Kim MJ, et al. Increased volume of lumbar surgeries for herniated intervertebral disc disease and costeffectiveness analysis: a nationwide cohort study. Spine 2018;43:585-93

24 The World Health Organization. World report on disability: chapter 4 rehabilitation. Available: https://www.spine.org/Documents/ ResearchClinicalCare/Guidelines/LumbarDiscHerniation.pdf [Accessed 16 Dec 2018].

25 Oosterhuis T, Costa LO, Maher CG. Rehabilitation after lumbar disc surgery. Cochrane Database Syst Rev 2014;3:CD003007.

26 Ostelo RWJG, de Vet HCW, Waddell G, et al. Rehabilitation following first-time lumbar disc surgery: a systematic review within the framework of the Cochrane collaboration. Spine 2003;28:209-18.

27 Danielsen JM, Johnsen R, Kibsgaard SK, et al. Early aggressive exercise for postoperative rehabilitation after discectomy. Spine 2000;25:1015-20.

28 Manniche C, Skall HF, Braendholt L, et al. Clinical trial of postoperative dynamic back exercises after first lumbar discectomy. Spine 1993;18:92-7.

29 McGregor AH, Probyn K, Cro S, et al. Rehabilitation following surgery for lumbar spinal stenosis. A cochrane review. Spine 2014;39:1044-54.

30 Abbott AD, Hedlund R, Tyni-Lenné R. Patients' experience postlumbar fusion regarding back problems, recovery and expectations in terms of the International classification of functioning, disability and health. Disabil Rehabil 2011;33:1399-408.

31 Archer K, Bird ML, Haug C, et al. Patients' experience and expectations of lumbar spine surgery for degenerative conditions: A qualitative study. Proceedings of the NASS 30th Annual Meeting. Spine J 2015;15:87S-267.

32 Rushton A, Heneghan NR, Heap A, et al. Patient and physiotherapist perceptions of rehabilitation following primary lumbar discectomy: a qualitative focus group study embedded within an external pilot and feasibility trial. BMJ Open 2017;7:e015878.
33 Rushton A, Staal JB, Verra M, et al. Patient journey following lumbar spinal fusion surgery (LSFS): protocol for a multicentre qualitative analysis of the patient rehabilitation experience (FuJourn). BMJ Open 2018;8:e020710.

34 Sibbern T, Bull Sellevold V, Steindal SA, et al. Patients' experiences of enhanced recovery after surgery: a systematic review of qualitative studies. J Clin Nurs 2017;26:1172-88.

35 Harden A, Thomas J, Cargo M, et al. Cochrane qualitative and implementation methods group guidance series-paper 5: methods for integrating qualitative and implementation evidence within intervention effectiveness reviews. J Clin Epidemiol 2018;97:70-8.

36 Moher D, Shamseer L, Clarke M, et al. Preferred reporting items fo systematic review and meta-analysis protocols (PRISMA-P) 2015 statement. Syst Rev 2015;4:1.

37 National Institute for Health Research. International prospective register of systematic reviews (Prospero). Available: https://www.crd. york.ac.uk/prospero/ [Accessed 10 Jan 2019].

38 Moher D, Liberati A, Tetzlaff J, et al. Preferred reporting items for systematic reviews and meta-analyses: the PRISMA statement. $J$ Clin Epidemiol 2009;62:1006-12.

39 Roland M, Morris R. A study of the natural history of back pain. Part I: development of a reliable and sensitive measure of disability in lowback pain. Spine 1983;8:141-4.

40 American Academy of Orthopaedic Surgeon. Ortholnfo: lumbar spinal stenosis. Available: https://orthoinfo.aaos.org/en/diseasesconditions/lumbar-spinal-stenosis [Accessed 22 Jan 2019]

41 The World Health Organization. Health promotion. Available: https:// www.who.int/topics/health_promotion/en/ [Accessed 1 Dec 2018].

42 The World Health Organization. Health promotion strategies. Available: http://www.searo.who.int/entity/healthpromotion/healthpromotion-strategies/en/ [Accessed 1 Dec 2018].

43 World Health Organization. The International classification of functioning, disability and health (ICF). Available: https://www. who.int/classifications/icf/icfbeginnersguide.pdf [Accessed $1 \mathrm{Dec}$ 2018]

44 Joos E, Peretz A, Beguin S, et al. Reliability and reproducibility of visual analogue scale and numeric rating scale for therapeutic evaluation of pain in rheumatic patients. J Rheumatol 1991;18:1269-70.

45 Ohnhaus EE, Adler R. Methodological problems in the measurement of pain: a comparison between the verbal rating scale and the visual analogue scale. Pain 1975;1:379-84.

46 Strong J, Ashton R, Chant D. Pain intensity measurement in chronic low back pain. Clin J Pain 1991;7:209-18.

47 Radloff LS. The CES-D scale: a self report depression scale for research in the general population. Appl Psychol Meas 1977:1:385-401.

48 Beck AT, Ward CH, Mendelson M, et al. An inventory for measuring depression. Arch Gen Psychiatry 1961;4:561-71.

49 Beck AT, Steer RA, Carbin MG. Psychometric properties of the Beck depression inventory: twenty-five years of evaluation. Clin Psychol Rev 1988;8:77-100.

50 Fairbank JC, Pynsent PB. The Oswestry disability index. Spine 2000;25:2940-53.

51 Ware JE, Sherbourne CD. The mos 36-item short-form health survey (SF-36). I. conceptual framework and item selection. Med Care 1992;30:473-83.

52 EuroQol Group. EuroQol--a new facility for the measurement of health-related quality of life. Health Policy 1990;16:199-208.

53 Pohlman KA, O'Beirne M, Thiel $\mathrm{H}$, et al. Development and validation of providers' and patients' measurement instruments to evaluate adverse events after spinal manipulation therapy. Eur J Integr Med 2014;6:451-66.

54 Zorzela L, Boon H, Mior S, et al. Serious adverse events associated with pediatric complementary and alternative medicine. Eur J Integr Med 2014;6:467-72.

55 Flemming $\mathrm{K}$, Booth $\mathrm{A}$, Hannes $\mathrm{K}$, et al. Cochrane qualitative and implementation methods group guidance series-paper 6: reporting guidelines for qualitative, implementation, and process evaluation evidence syntheses. J Clin Epidemiol 2018;97:79-85.

56 Noyes J, Booth A, Cargo M, et al. Cochrane qualitative and implementation methods group guidance series-paper 1: introduction. J Clin Epidemiol 2018;97:35-8.

57 Norman GR, Streiner DL. Biostatistics: the bare essentials. 3. Hamilton: BD Decker, 2008

58 World Health Organization. Community-Based rehabilitation. Available: https://www.who.int/disabilities/cbr/en/ [Accessed 1 Dec 2018]

59 Follett KA, Dirks BA. Etiology and evaluation of the failed back surgery syndrome. Neurosurg Q 1993;3:40. 
60 McGowan J, Sampson M, Lefebvre C. An evidence based checklist for the peer review of electronic search strategies (press EBC). Evid Based Libr Inf Pract 2010;5:149-54.

61 Sampson M, McGowan J, Cogo E, et al. An evidence-based practice guideline for the peer review of electronic search strategies. $J$ Clin Epidemiol 2009;62:944-52.

62 Centre EfPaPlaC. EPPI-Reviewer 4 software. Available: eppi.ioe.ac. uk [Accessed 20 Nov 2019].

63 Cieza A, Brockow T, Ewert T, et al. Linking health-status measurements to the International classification of functioning, disability and health. J Rehabil Med 2002;34:205-10.

64 Cieza A, Fayed N, Bickenbach J, et al. Refinements of the ICF linking rules to strengthen their potential for establishing comparability of health information. Disabil Rehabil 2019;41:574-83.

65 Cieza A, Geyh S, Chatterji S, et al. Icf linking rules: an update based on lessons learned. J Rehabil Med 2005;37:212-8.

66 Scottish Intercollegiate Guidelines Network (SIGN). Critical appraisal notes and checklists,, 2019. Available: https://www.sign.ac.uk/ checklists-and-notes.html [Accessed 1 Feb 2019].

67 Lockwood C, Munn Z, Porritt K. Qualitative research synthesis: methodological guidance for systematic reviewers utilizing metaaggregation. Int J Evid Based Healthc 2015;13:179-87.
68 Pluye P, Gagnon M-P, Griffiths F, et al. A scoring system for appraising mixed methods research, and concomitantly appraising qualitative, quantitative and mixed methods primary studies in mixed studies reviews. Int J Nurs Stud 2009;46:529-46.

69 Higgins JPT, Thompson SG. Quantifying heterogeneity in a metaanalysis. Stat Med 2002;21:1539-58.

70 Slavin RE. Best evidence synthesis: an intelligent alternative to metaanalysis. J Clin Epidemiol 1995;48:9-18.

71 Follmann D, Elliott P, Suh I, et al. Variance imputation for overviews of clinical trials with continuous response. J Clin Epidemiol 1992;45:769-73.

72 Abrams KR, Gillies CL, Lambert PC. Meta-Analysis of heterogeneously reported trials assessing change from baseline. Stat Med 2005;24:3823-44.

73 Thomas J, Harden A. Methods for the thematic synthesis of qualitative research in systematic reviews. BMC Med Res Methodol 2008;8:45.

74 Noyes J, Booth A, Flemming K, et al. Cochrane Qualitative and Implementation Methods Group guidance series-paper 3: methods for assessing methodological limitations, data extraction and synthesis, and confidence in synthesized qualitative findings. J Clin Epidemiol 2018;97:49-58. 